\title{
Vibration pattern related to transverse cracks in rotors
}

\author{
Nicolò Bachschmid* and Ezio Tanzi \\ Politecnico di Milano, Dipartimento di Meccanica, Via La Masa, 34, I-20158, Milano, Italy
}

\begin{abstract}
A method for calculating the breathing behavior of transverse cracks of different types in rotating shafts is described. Thermal effects are included. Some results in terms of vibration excitation related to different shapes of cracks are presented.
\end{abstract}

Keywords: Cracks, breathing, vibrations, rotors

\section{Introduction}

The dynamical behavior of horizontal heavy rotors presenting a transverse crack has been studied by many researchers, (see e.g. the overview of Dimoragonas [3]). The behavior can be described in the following way: during one rotation of the rotor, the crack moves from the upper position in which the statical bending moment forces the crack to be "closed" to the opposite position in which the crack is forced to be "open". The gradually opening and closing of the crack is called the breathing mechanism. The stiffness of the rotor with the open crack is smaller with respect to the closed crack situation, in which the stiffness equals the value of the uncracked shaft. Therefore the stiffness changes periodically during one rotation, and correspondingly also the statical deflection due to the weight (and to the bearing alignment conditions). The periodical deflection and the periodical stiffness can both be expanded in a Fourier series, of which the most important components are the $1 \mathrm{x}, 2 \mathrm{x}$ and $3 \mathrm{xrev}$. components. It can be shown that the same forces which excite the statical deflection components, excite also the dynamical vibrations, when the shaft is rotating at higher speeds. The vibration pattern of a cracked shaft is determined by the relative weight of the three harmonic components of the vibration which are related to the corresponding

*Corresponding author. Tel.: +3902 2399 8426; Fax: +39 02 2399 8492; E-mail: nicolo.bachschmid@polimi.it. components of the stiffness of the shaft, and therefore to the breathing mechanism.

Almost all studies about cracks are based on the fracture mechanics approach, which allows to calculate the flexibility of the open crack situation, but does not give any hints for the breathing mechanism of cracks in rotating shafts. Some authors have analyzed the breathing mechanism of vibrating non rotating beams, see e.g. Abraham [1]. Many authors represent the breathing of cracks in rotating beams with a simple analytical function, but the form of this function determines the vibration pattern. Moreover the breathing mechanism and the vibration behavior of the cracked rotor is influenced by thermal stresses which can arise during thermal transients when the operating conditions of the machine are changing. This thermal sensitivity of cracked rotors is well known from field experiences, and has been used for detecting the presence of cracks in rotors operating in power plants.

In this paper a method for calculating the breathing behavior of cracks including thermal effects is presented which has been applied to cracks of different shapes in rotors in order to highlight the related differences in their dynamical behaviors.

\section{Fault modelling and identification}

Figure 1 shows the comparison of the $1 \mathrm{xrev}$. components of the vibration of the rotor of a steam turbine dur- 

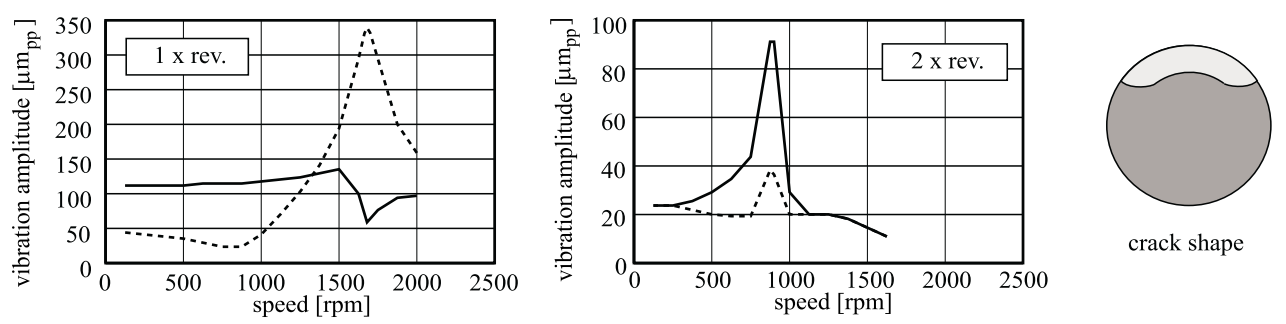

Fig. 1. 1xrev. and 2xrev component vs. speed, continuous line corresponds to cooling transient and dotted line corresponds to heating transient, and related crack shape.

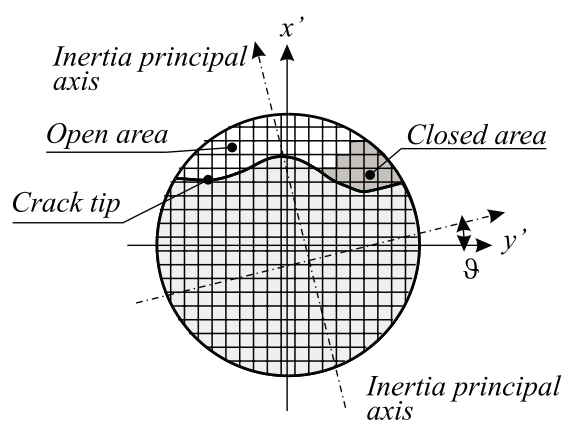

Fig. 2. Cracked cross section.

ing a slow run down in two different thermal situations. The continuous line represents the cracked rotor during a cooling transient when the thermal stresses open the crack: the rotating bow due to the open crack (recognisable at low speed) is partially compensated by some balancing masses as can be seen when approaching the critical speed.

The dotted line represents the behavior during the heating transient when the thermal stresses close the crack: the bow is strongly reduced and the typical peak of resonance due to the balancing masses appears in correspondence of the critical speed. Figure 1 shows also the behavior of the $2 x r e v$. component of the vibration: considering the run down during the cooling transient (continuous line) the excitation of the $2 x r e v$ vibrations is higher with respect to the same run-down during the heating transient (this can be seen especially when crossing the secondary critical speed). The vibrations were measured close to the position of the crack.

Both figures show how strongly the breathing behavior (and correspondingly the vibration pattern) is influenced by the thermal stresses.

Also the profile of the crack which caused above described behavior, as defined by U.S. test, is represented in Fig. 1. The shown results were obtained during a series of test-runs in the test cell of the turbine manufacturer, to which the authors partecipated.

\section{The proposed crack model}

The crack model, a draft version of which was already presented by Bachschmid [2], is composed by a simplified thermal model, which considers an infinite cylinder and axial symmetrical temperature and stress distributions, and by a simplified model of the breathing mechanism, which depends of the statical bending moment stresses and of the thermal stresses. In the points of the cracked area where the stresses are compressive, there contact occurs between the two faces of the crack; where the stresses are instead tensile, there no contact occurs. In this way the open and closed parts of the cracked area are determined in the different angular positions. The procedure is obviously roughly approximated since the actual stress distribution over the cracked cross section due to bending moment is not at all linear (as assumed by the simplified model) and the superposition of thermal stresses might not be correct (due to the non-linearity in the behavior of the cracked area).

\subsection{Thermal behaviour}

To determine the temperature distribution, the equation of the thermal exchange is used in the case of axial-symmetry and of an infinite cylinder:

$$
\frac{\rho c_{p}}{k} \frac{\partial T}{\partial t}=\frac{1}{r} \frac{\partial}{\partial r} r \frac{\partial T}{\partial r}
$$

Where $\rho$ is the density, $c_{p}$ the specific heat, $k$ the conduction coefficient, $r$ the radial coordinate, $T$ the generic temperature of the section and the time. As boundary condition a temperature gradient $\Delta T / \Delta t$ has been imposed on the external surface:

$$
\begin{aligned}
& \left.\frac{\partial T}{\partial r}\right|_{\text {rint }}=0 \\
& T_{\text {rext }}(t+\Delta t)=T_{\text {rext }}(t)+\Delta T
\end{aligned}
$$

Finite difference method has been used for solving Eq. (1). This leads to a system of the following type: 

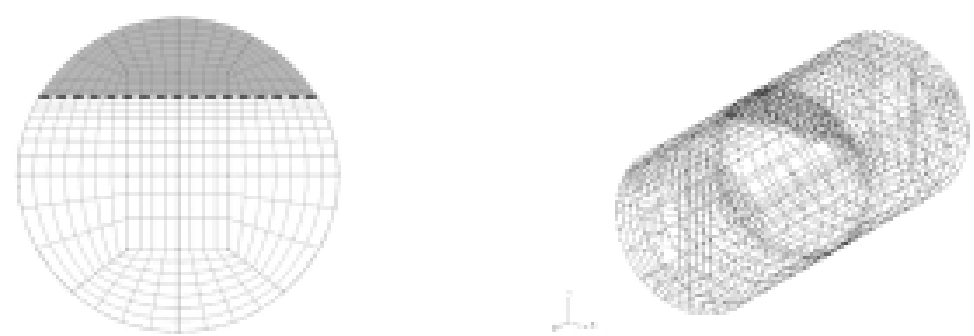

Fig. 3. Mesh of the section and isometric view of the model with a crack of $25 \%$. The crack tip is indicated by the dashed line.
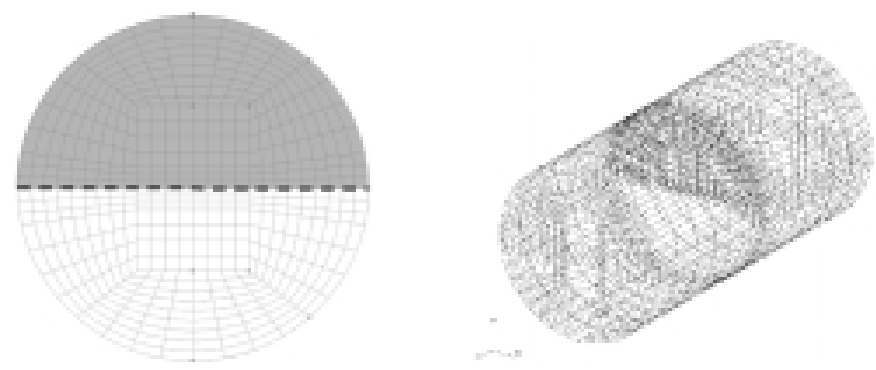

Fig. 4. Mesh of the section and isometric view of the model with a crack of 50\%. The crack tip is indicated by the dashed line.
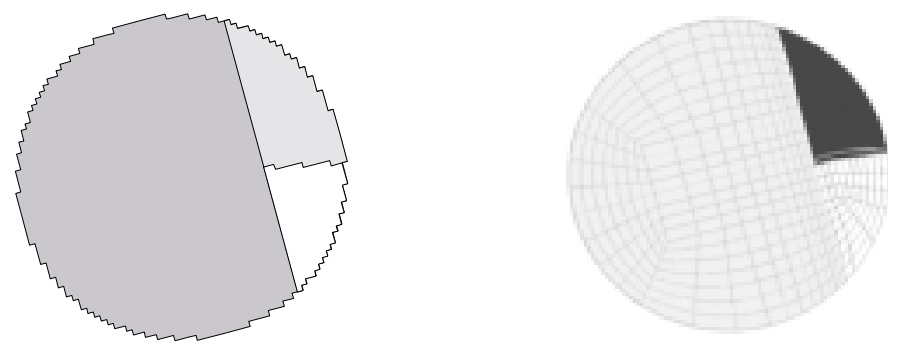

Fig. 5. Comparison between the results of the simplified model and the 3D f.e. model for a $25 \%$ depth crack, rotation of 75 , simplified model (left), 3D model (right).

$$
\{T(t+\Delta t)\}=[A]\{T(t)\}
$$

where the matrix $[A]$ has constant coefficients, $\{T(t+$ $\Delta t)\}$ and $\{T(t)\}$ are the vectors of the temperatures in the radial coordinates determined over the section. By iterating Eq. (3) with time step $\Delta t$, it is possible to determine the temperature distribution in each point of the section as function of the time. The axial stress distribution corresponding to the temperature distribution is given by:

$$
\sigma=\frac{\alpha T}{1-v}\left(T_{m}-T(r)\right)
$$

where $\alpha$ is the linear thermal expansion coefficient, $E$ the Young's modulus, $\nu$ the Poisson's coefficient, $T(r)$ the temperature at the radial coordinate $r$ and $T_{m}$ the average section temperature. The effect of the centrifugal forces on the axial stresses has been neglected.

\subsection{Breathing mechanism}

In the following, the different steps for modelling the breathing behavior, including thermal effects, are illustrated:

a) In correspondence of the cracked section, the cross sectional area $A$ is divided into small area elements $d A=d x d y$ according to a rotating reference system (fixed on the rotor) $x^{\prime} y^{\prime}$ (Fig. 2);

b) The bending moment $M$ due to the weight and the bearing alignment conditions of the rotor is calculated in correspondence of the cracked section. 

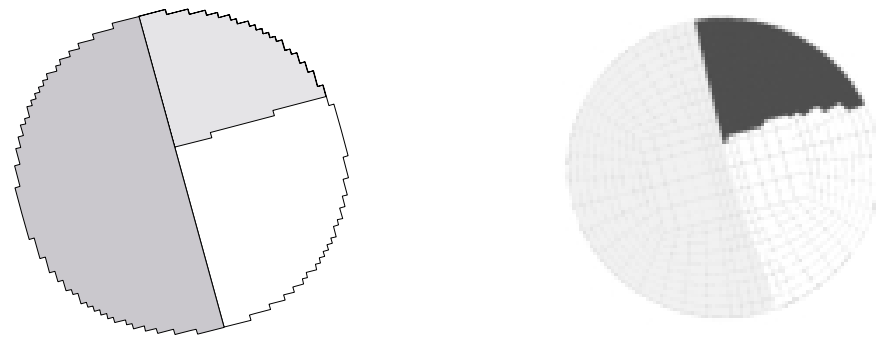

Fig. 6. Comparison between the results of the simplified model and the 3D f.e. model for a $50 \%$ depth crack, rotation of 79 , simplified model (left), 3D model (right).
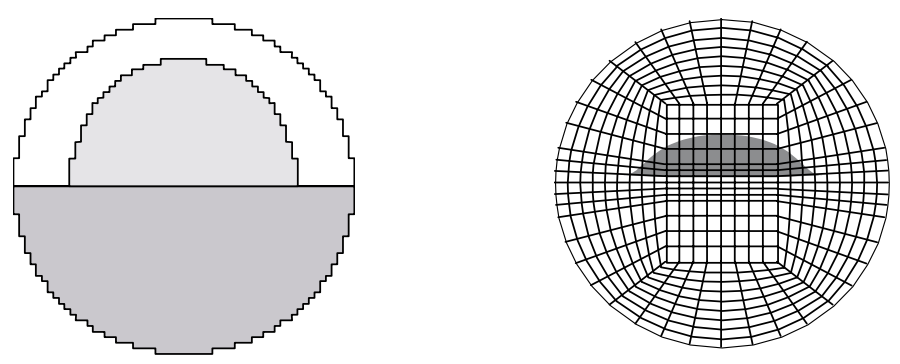

Fig. 7. Thermal load only - Negative gradient. Simplified model (left), 3D model (right).
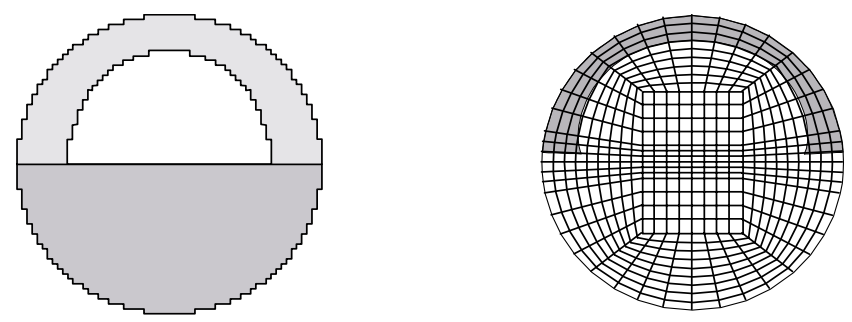

Fig. 8. Thermal load only - Positive gradient. Simplified model (left), 3D model (right).

c) One revolution $\left(360^{\circ}\right)$ of the shaft is divided in 128 parts: in each position following calculations are performed:

d) An iterative procedure is started in order to define the open and closed sections of the cracked area, the position of the centre of gravity $G$ of the closed surface, the position of the main axes of inertia (angle $\vartheta$ ) with origin in $G$, the second area moments with respect to the main axes and the moments $M_{x p}, M_{y p}$ due to the thermal stress distribution;

$\left.d_{1}\right)$ Initially the main axes $\left(x^{\prime} m, y^{\prime} m\right)$ are considered to be coincident with the rotating crack axes $\left(x^{\prime} y^{\prime}\right)$; the stresses due to bending moment are calculated (with the assumed $x^{\prime} y^{\prime}$ main axes), and the thermal stresses are then added in each point; $d_{2}$ ) Now the stress distribution is known over the cross section and the sign of the stress can be checked in each point of the cracked area: ' + ' means tension and therefore we have no contact forces in this point (the crack area element is "open"), '-' means compression and therefore we have contact forces (the crack area element is "closed"). The open and closed area sections have been determined.

$d_{3}$ ) The surface gravity center of the total area (formed by the uncracked area plus the closed cracked area) can be calculated.

$d_{4}$ ) The second moments of area can now be calculated with respect to reference system $x^{\prime} y^{\prime}$ with origin in $G$ and the angular position $\vartheta$ of the main axes of inertia $x^{\prime} m, y^{\prime} m$ can be found; 

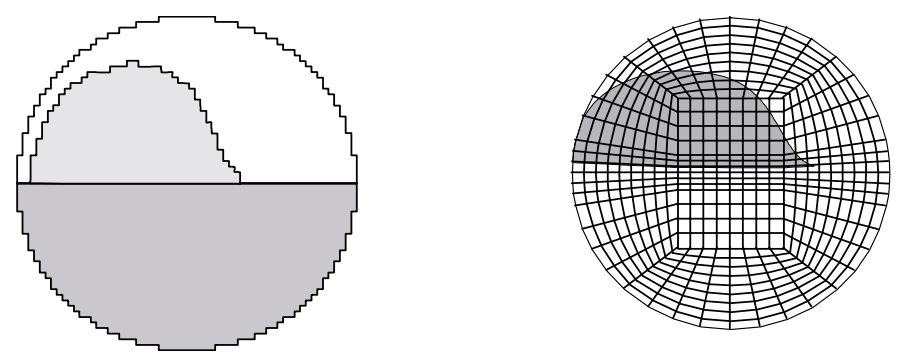

Fig. 9. Thermal and mechanical load - Negative gradient - Angular position 120, simplified model (left) 3D model (right).
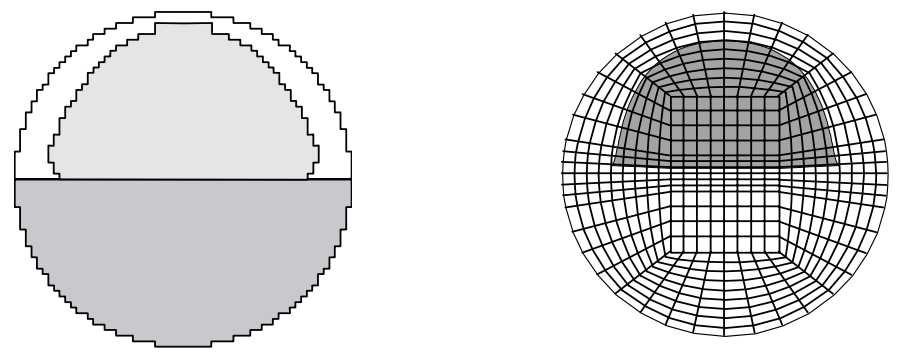

Fig. 10. Thermal and mechanical load - Negative gradient - Angular position $180^{\circ}$, simplified model (left) 3D model (right).

$\left.d_{5}\right)$ Now the procedure from $\left(d_{1}\right)$ to $\left(d_{4}\right)$ is repeated until $\vartheta$ converges to a stable value;

e) At this point the position of the main axis and the second area moments and $M_{x p}, M_{y p}$ are known. The second area moment $J_{x}, J_{y}$ and $J_{x y}$ with respect to the fixed reference frame $(x y)$ and the components of the moments due to the thermal stress distribution $\left(M_{x}, M_{y}\right)$ with respect to the same reference frame are calculated. This will be repeated for each angular position of the shaft.

f) A Fourier analysis over 128 values of $J_{x}, J_{y}$ and $J_{x y}$ and $M_{x}$ and $M_{y}$ is carried out, and the mean values $J_{x m}, J_{y m}, J_{x y m}$ and their first three harmonic components $\left(J_{x 1}, J_{x 2}, J_{x 3}\right.$ and so on) are extracted.

\section{Breathing mechanism validation}

The breathing mechanism calculated with the described simplified approach, has been validated with numerical results obtained with a 3D model of a cracked cylindrical beam, clamped at one end and loaded mechanically at the other end with a rotating load. Also temperature gradients have been imposed to the outer surface of the cylindrical specimen.

Generally a rather good agreement has been found between the simplified linear model and the 3D non linear model. Figure 3 shows the mesh used for a cracked cylinder with a $25 \%$ relative depth, and Fig. 4 that one of a $50 \%$ relative depth.

Figures 5 and 6 show the comparison of 3D results in the position where the rotor (or the load) is rotated by $75^{\circ}$, for the $25 \%$ and the $50 \%$ depth crack, with the simplified model results: a very good agreement has been found as also in all the other positions. The dark areas are closed, the white areas of the crack are open.

When the thermal transient (negative or positive temperature gradient on the outer surface) is applied to the specimen, the agreement between the results of the two models is excellent except in the vicinity of the cracked section, where significant differences between the two model results appear, mainly for the negative temperature gradient. This is shown in Fig. 7 for the negative gradient and in Fig. 8 for the positive gradient. Similar results have been found for the smaller crack $(25 \%$ depth).

When the thermal transient is superposed to the mechanical loading, then a better agreement is found in general. This is shown in Fig. 9 where the angular position of $120^{\circ}$ is represented in case of negative temperature gradient and in Fig. 10 for the angular position of $180^{\circ}$.

Similar results are found in other angular positions and for the smaller crack also. The results have been obtained in following conditions: 


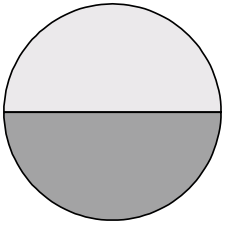

rectilinear $50 \%$

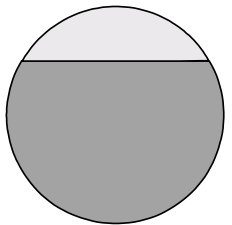

rectilinear $25 \%$

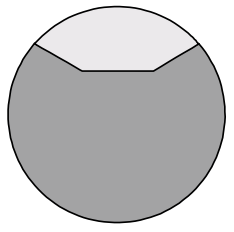

three-linear crack

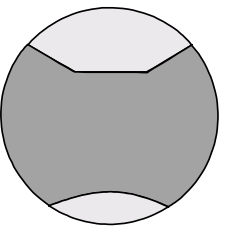

double crack

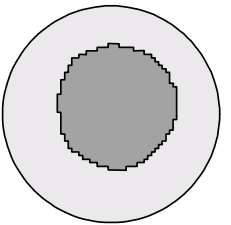

annular crak

Fig. 11. Different shapes of crack.

Table 1

Adimensional second moments of area represented as 1st, 2nd and 3rd harmonic components referred to the second moment of area of the uncracked cross section

\begin{tabular}{llccccc}
\hline & & $25 \%$ & $50 \%$ & Three-linear & Double & Annular \\
\hline 1xrev. & Jx & $1.28 \mathrm{E}-01$ & $2.48 \mathrm{E}-01$ & $1.25 \mathrm{E}-01$ & $8.18 \mathrm{E}-02$ & $3.63 \mathrm{E}-02$ \\
& Jy & $2.35 \mathrm{E}-01$ & $4.40 \mathrm{E}-01$ & $2.33 \mathrm{E}-01$ & $1.39 \mathrm{E}-01$ & $7.37 \mathrm{E}-02$ \\
& Jxy & $1.14 \mathrm{E}-01$ & $1.72 \mathrm{E}-01$ & $1.13 \mathrm{E}-01$ & $6.72 \mathrm{E}-02$ & $3.64 \mathrm{E}-02$ \\
2 xrev. & Jx & $1.23 \mathrm{E}-01$ & $2.07 \mathrm{E}-01$ & $1.32 \mathrm{E}-01$ & $1.62 \mathrm{E}-01$ & $2.11 \mathrm{E}-03$ \\
& Jy & $8.97 \mathrm{E}-02$ & $4.64 \mathrm{E}-02$ & $9.90 \mathrm{E}-02$ & $1.47 \mathrm{E}-01$ & $6.18 \mathrm{E}-03$ \\
& Jxy & $8.78 \mathrm{E}-02$ & $4.22 \mathrm{E}-02$ & $9.76 \mathrm{E}-02$ & $1.46 \mathrm{E}-01$ & $6.08 \mathrm{E}-03$ \\
$3 x$ xrev. & Jx & $6.69 \mathrm{E}-02$ & $3.11 \mathrm{E}-02$ & $8.46 \mathrm{E}-02$ & $4.23 \mathrm{E}-02$ & $1.90 \mathrm{E}-03$ \\
& Jy & $1.26 \mathrm{E}-02$ & $1.41 \mathrm{E}-02$ & $1.82 \mathrm{E}-02$ & $4.85 \mathrm{E}-03$ & $2.27 \mathrm{E}-04$ \\
& Jxy & $2.11 \mathrm{E}-02$ & $1.25 \mathrm{E}-02$ & $2.97 \mathrm{E}-02$ & $8.63 \mathrm{E}-03$ & $2.47 \mathrm{E}-04$ \\
\hline
\end{tabular}

- Specimen dimensions: diameter $25 \mathrm{~mm}$, length $50 \mathrm{~mm}$;

- Applied loads: bending $10 \mathrm{Nm}$;

- Material standard steel, Temperature gradient \pm $100^{\circ} \mathrm{C} / \mathrm{min}$;

- Thermal stresses calculated after $5 \mathrm{sec}$.

All these results seem to confirm the validity of the proposed simplified method: only during thermal transients and in particular conditions some of the accuracy is lost, but the enormous saving of d.o.f.s. and calculation time justifies the choice of the simplified model. The mechanical load is always present, sometimes also the thermal load, but thermal load alone does never exist in real machines.

\section{Results obtained with different shapes of cracks}

Figure 11 shows the different shapes of crack which have been analysed.

The effects of the different breathing behaviours in the presence or not of thermal transients have been calculated and are reported in Tables 1 and 2, where the results are represented as 1st, 2nd and 3rd harmonic components of the second moments of area referred to the second moment of area of the uncracked cross section. Since the stiffness matrix components depend linearly on the second moments of area, and the dis- placements (statical deflections and vibrations) depend on the stiffness matrices, the second moments of area give an idea about the expected vibration pattern.

Since it is difficult to argue from the Tables 1 and 2 which are the changes in vibration behavior due to the differences in crack shapes, because the behavior is influenced by the combination of the 3 values of the second moments of area, in some cases the vibrations which could be measured in a bearing of real turbogenerator rotor represented in Fig. 12 have been calculated and are reported in Figs 13, 14, 15 and 16.

It must be noted that the thermal bow which influences strongly the 1xrev. vibration component (see Fig. 1) is not considered in this analysis. From the analysis of Tables 1 and 2 and from the diagrams of Figs 13, 14, 15 and 16 following conclusions can be drawn.

a) Comparing the two rectilinear cracks, the $50 \%$ depth to the $25 \%$ depth, it can be seen that the $1 \mathrm{xrev}$ component is higher for the deeper crack with respect to the smaller crack, and the $2 \mathrm{xrev}$. component seems to be instead smaller for the deeper crack with respect to the smaller crack. The thermal transient sensitivity is similar for the $1 \mathrm{xrev}$. component although the smaller crack is more sensitive to both negative and positive gradients. The sensitivity to thermal effects of the $2 \mathrm{xrev}$. component is much higher for the smaller 
Table 2

Effect of positive $(+\Delta t)$ and negative $(-\Delta t)$ temperature gradients on the second moments of area of Table 1

\begin{tabular}{|c|c|c|c|c|c|c|c|c|c|c|c|}
\hline & & \multicolumn{2}{|c|}{$25 \%$} & \multicolumn{2}{|c|}{$50 \%$} & \multicolumn{2}{|c|}{ Three-Linear } & \multicolumn{2}{|c|}{ Double } & \multicolumn{2}{|c|}{ Annular } \\
\hline & & $+\Delta t$ & $-\Delta t$ & $+\Delta t$ & $-\Delta t$ & $+\Delta t$ & $-\Delta t$ & $+\Delta t$ & $-\Delta t$ & $+\Delta t$ & $-\Delta t$ \\
\hline \multirow[t]{3}{*}{ 1xrev. } & $\mathrm{Jx}$ & $-44 \%$ & $-56 \%$ & $20 \%$ & $-33 \%$ & $-43 \%$ & $-54 \%$ & $-23 \%$ & $-49 \%$ & $95 \%$ & $-35 \%$ \\
\hline & Jy & $-13 \%$ & $-35 \%$ & $-5 \%$ & $-22 \%$ & $-13 \%$ & $-32 \%$ & $19 \%$ & $-32 \%$ & $10 \%$ & $-9 \%$ \\
\hline & Jxy & $-58 \%$ & $-54 \%$ & $-15 \%$ & $-31 \%$ & $-53 \%$ & $-54 \%$ & $-34 \%$ & $-49 \%$ & $27 \%$ & $-37 \%$ \\
\hline \multirow[t]{3}{*}{ 2xrev. } & $\mathrm{JX}_{\mathrm{X}}$ & $-94 \%$ & $18 \%$ & $-75 \%$ & $-61 \%$ & $-84 \%$ & $9 \%$ & $-86 \%$ & $34 \%$ & $66 \%$ & $40 \%$ \\
\hline & Jy & $37 \%$ & $23 \%$ & $82 \%$ & $75 \%$ & $30 \%$ & $24 \%$ & $4 \%$ & $8 \%$ & $21 \%$ & $-12 \%$ \\
\hline & Jxy & $-27 \%$ & $45 \%$ & $122 \%$ & $96 \%$ & $-18 \%$ & $39 \%$ & $-38 \%$ & $27 \%$ & $29 \%$ & $-41 \%$ \\
\hline \multirow[t]{3}{*}{$3 x r e v}$. & $\mathrm{Jx}$ & $-92 \%$ & $-48 \%$ & $251 \%$ & $85 \%$ & $-84 \%$ & $-60 \%$ & $-82 \%$ & $-47 \%$ & $-48 \%$ & $55 \%$ \\
\hline & Jy & $257 \%$ & $30 \%$ & $108 \%$ & $-51 \%$ & $143 \%$ & $27 \%$ & $530 \%$ & $-37 \%$ & $-50 \%$ & $-4 \%$ \\
\hline & Jxy & $52 \%$ & $13 \%$ & $-91 \%$ & $13 \%$ & $34 \%$ & $-6 \%$ & $264 \%$ & $41 \%$ & $10 \%$ & $183 \%$ \\
\hline
\end{tabular}

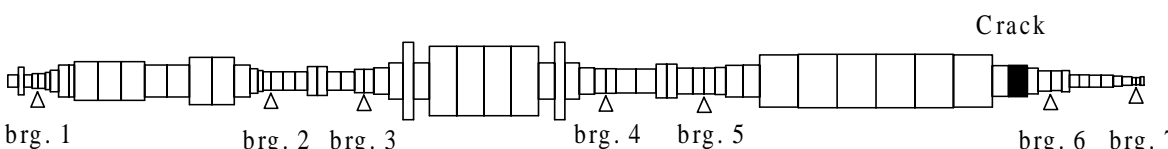

Fig. 12. Rotor model.

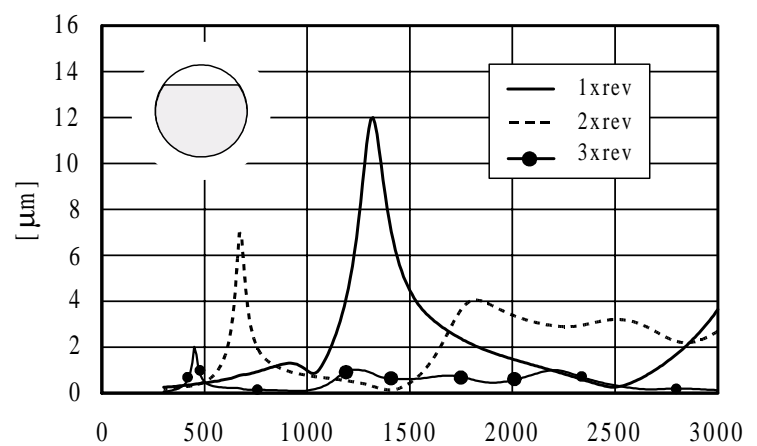

Fig. 13. Vertical vibration amplitude vs. speed in bearing 6: $30 \%$ crack depth.

cracks: in correspondence of the negative gradient all components increase and in correspondence of the positive gradient the mean value decreases. This does not occur in the deeper crack.

b) Three-linear crack and elliptical crack.

The tree-linear crack is compared with a rectilinear crack (25\% depth) which has a similar cracked area. The vibration pattern is very similar as can be seen also from Fig. 14 which refer to elliptical cracks with $30 \%$ depth. The behaviors with and without thermal effects are very similar. Only smaller differences are found in the $2 x r e v$ and $3 x$ rev components.

c) Double crack

This crack is composed by the previously shown three-linear or elliptical crack and by a smaller crack on the opposite side. The results are compared with those of the three linear crack alone. As can be seen from Table1, the 1xrev. excitation

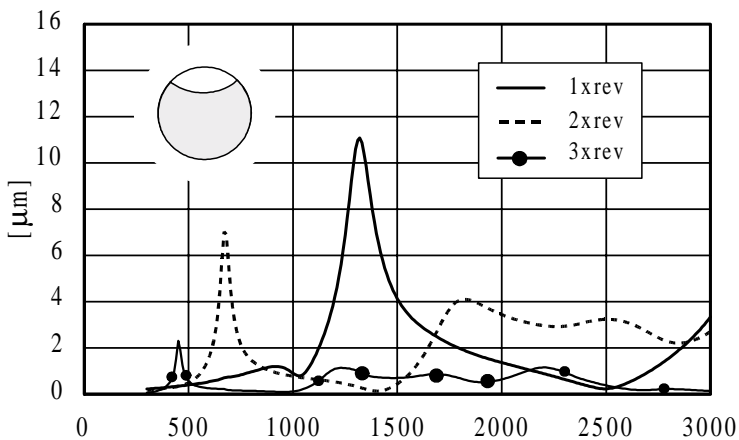

Fig. 14. Vertical vibration amplitude vs. speed in bearing 6: $30 \%$ elliptical crack.

is smaller, the $2 x r e v$. excitation is similar and the $3 x$ rev. is again smaller in the double crack, with respect to the three-linear crack. This can be seen also comparing the double crack behavior (Fig. 15) to the single crack behavior (Fig. 14). Also the thermal sensitivity of the 2xrev component is a little higher in the double crack.

d) Full annular crack

As can be seen from Table 1 the $1 x r e v ., 2 x r e v$. and still more the $3 x r e v$. excitations are rather small compared to other types of cracks. This behaviour could be expected, because an axialsymmetrical crack does not excite any harmonic component. As regards the thermal sensitivity this crack type presents a weak sensitivity to the temperature transients, although the percentage change are rather high, but it is interesting to note that the behaviour is opposite with respect to other types of cracks: the negative gradient lowers the 


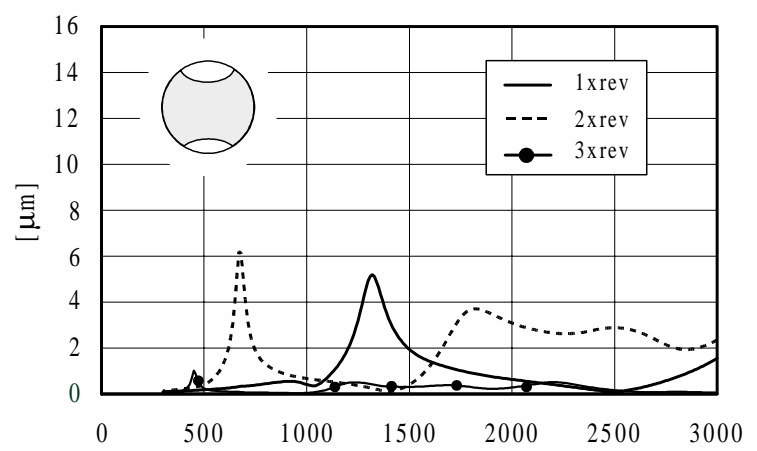

Fig. 15. Vertical vibration amplitude vs. speed in bearing 6: double crack.

2xrev. and increases the $3 x$ rev. excitation and the positive gradient increase the $2 x r e v$. excitation and lowers the $3 x r e v$. In other types of cracks exactly the opposite occurs.

e) $30 \%$ rectilinear slot (or fully open crack)

Figure 15 shows that only 2xrev. vibration components are present and that its amplitude is roughly double with respect to the $30 \%$ breathing crack.

\section{Conclusions}

A method for calculating the breathing behaviour of transverse cracks in rotors has been presented. The approach accounts also for the presence of thermal stresses which influence strongly the breathing behaviours.

The method has been validated by comparing the results with those obtained with a rather refined 3D non linear model, in several different conditions.

The method has then be used for calculating and comparing characteristic behaviours of different shapes of cracks. As far as the authors know, the proposed method is the only one which allows to calculate these behaviours in a simple, approximate way, without the

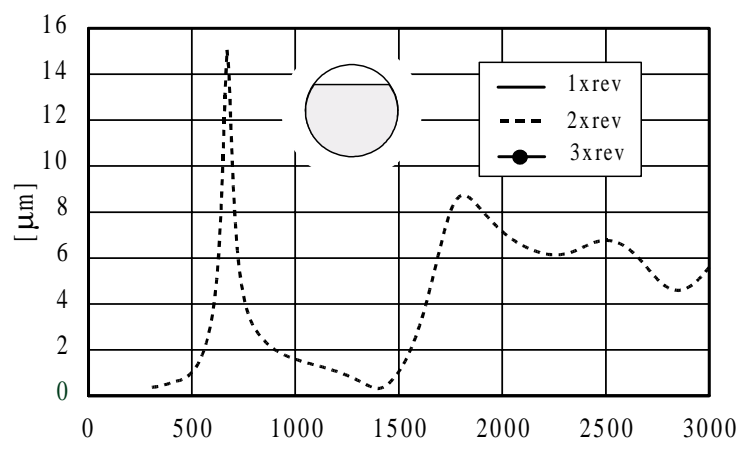

Fig. 16. Vertical vibration amplitude vs. speed in bearing 6: $30 \%$ slot/open crack.

need of cumbersome and time consuming 3D calculations.

The results of the breathing mechanism analysis can be directly introduced in a code for cracked rotor dynamical behavior simulation, which has been developed by the authors.

\section{Acknowledgements}

The financial support of MIUR "Tecniche di identificazione di malfunzionamenti di sistemi meccanici basate sull'analisi del comportamento dinamico" and MURST "Identificazione di malfunzionamenti di sistemi meccanici” is gratefully acknowledged.

\section{References}

[1] O.N.L. Abraham and J.A. Brandon, The modelling of the opening and closure of a crack, ASME Journal of Vibration and Acoustics 117 (July 1995), 370-377.

[2] N. Bachschmid and A. Collina, Cracked rotors: diagnosis and computational methods, PACAM III Proceedings, Sao Paulo, Brasil, January 4-8, 1993, pp. 235-239.

[3] A.D. Dimarogonas, Vibration of cracked structures: a state of the art review, Engineering Fracture Mechanics 55(5) (1996), $831-857$. 

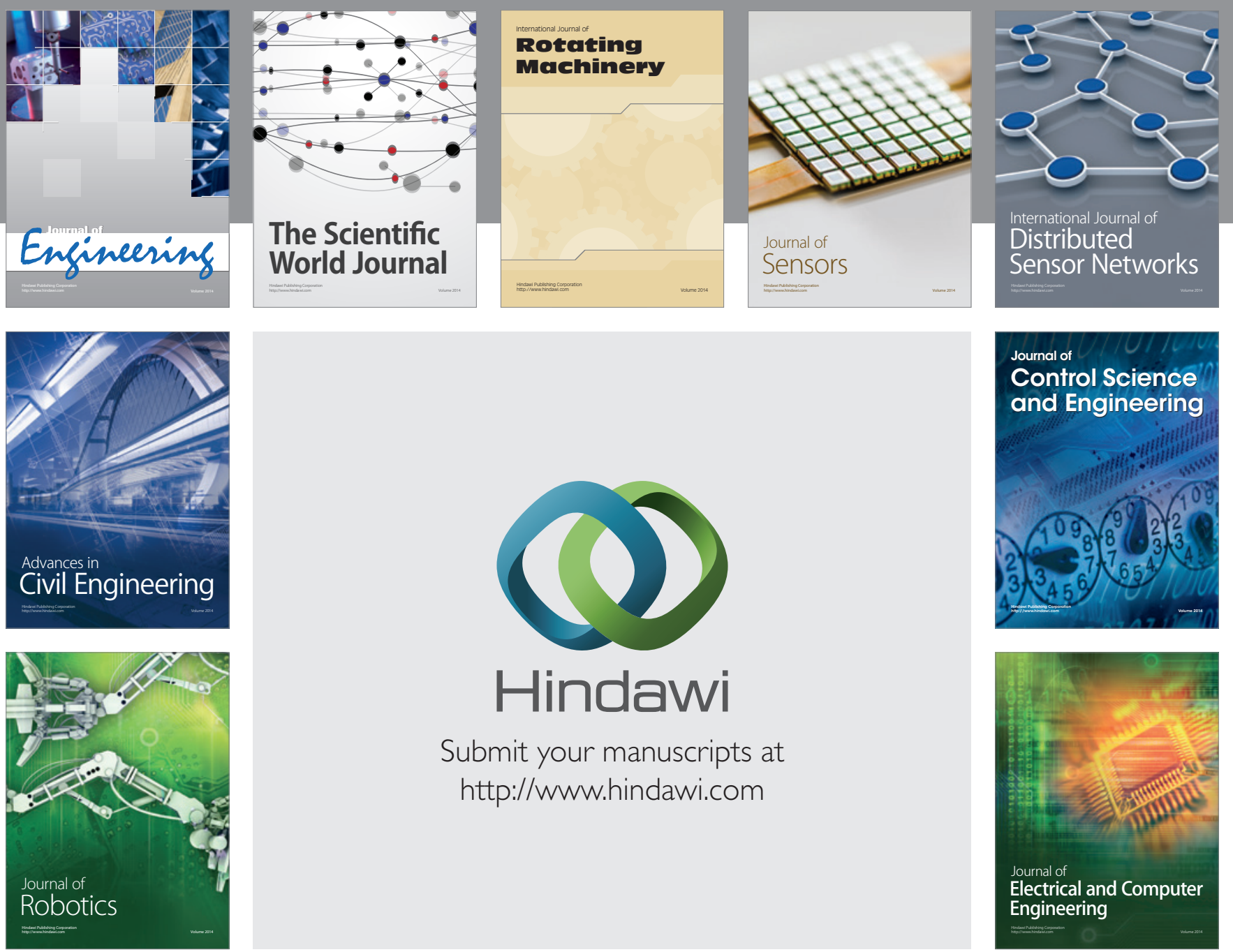

Submit your manuscripts at

http://www.hindawi.com
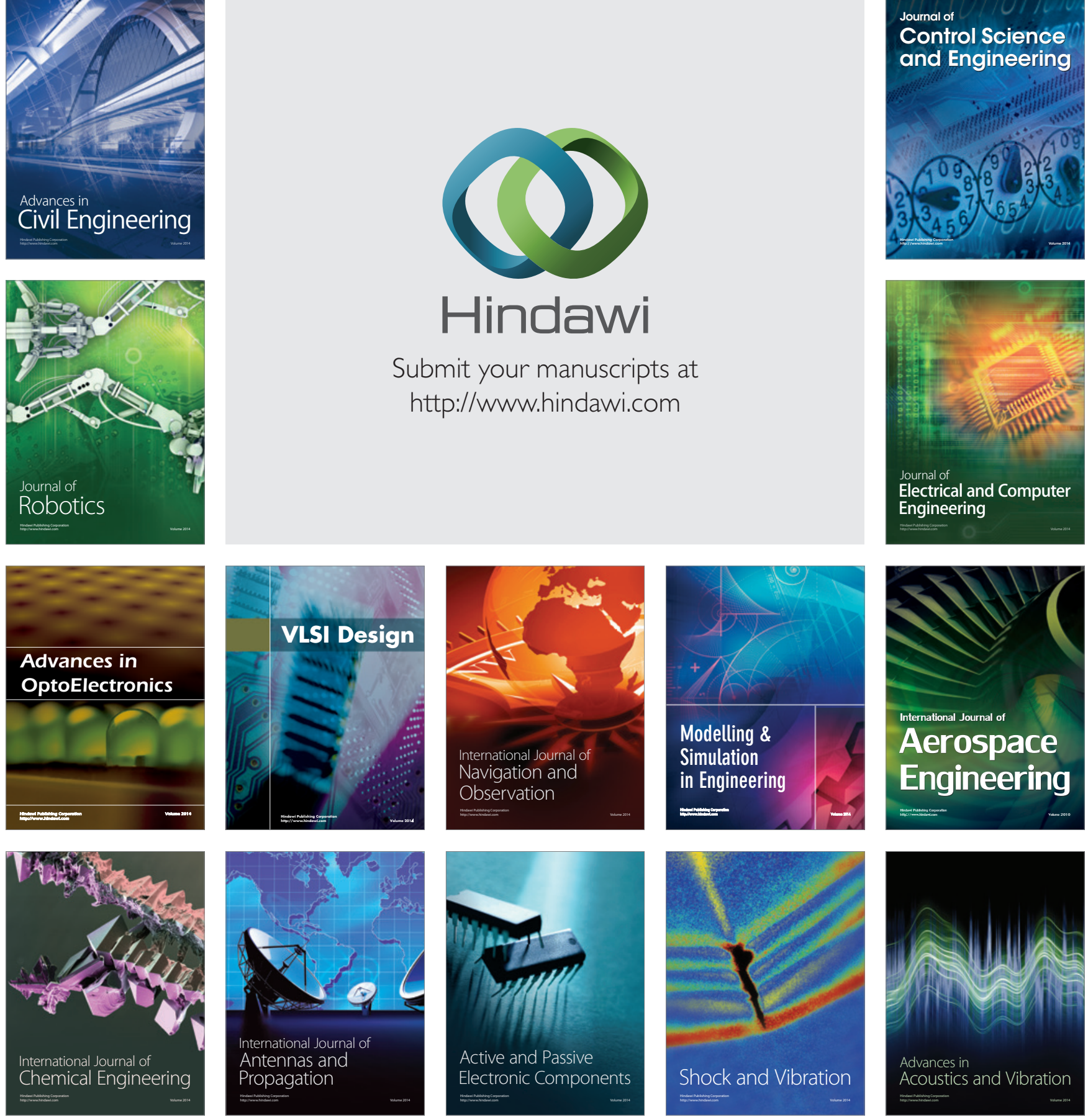